Short Communication

\section{A new applied protocol concerning COVID-19's pandemic control: "Outdoor Access Approach" remodel of living for ensuring better ventilation}

\author{
Nasser Alidadi* \\ Department of Large Animal Health and Internal Medicine, Faculty of Veterinary Medicine, University of \\ Tehran, Tehran, Iran
}

\section{Abstract}

COVID-19 is a mucoso-respiratory highly contagious disease that has leaded to a tremendous global pandemic wide spreading throughout nations of all continents with successive waves of high morbidities and mortalities. However, several independent vaccine production projects are working ahead for combatting the pandemic, but it is obvious they cannot create a sufficient umbrella that could protect billions of humans in a short term. Indeed, the current approved protocols including frequent cleaning of hands, social distancing and covering face mask are disappointing for their claimed capabilities to efficiently control of the pandemic. As they failed to highlight the critical determining role of air refreshing into indoors that 90 percent of infected people at least involved in enclosed spaces. Instead, Outdoor Access Approach (OAA) can be promising according veterinary medical successful experiences in control of air-borne contagious diseases as control of highly pathogenic avian influenza to become a global pandemic (One Health integration). Along with the above-mentioned protocols. Adaptation of all aspects of ordinary life activities in human societies to guarantee an efficient fresh air flow into enclosed spaces and prevention of the most dangerous air stagnation in them is the core stone of the OAA. This article discusses practical suggestions to attain such situation which can provide a kind of symbiosis with COVID-19' infection major threatening.

\section{More Information}

*Address for Correspondence: Nasser Alidadi, DVM, DVSc, Department of Large Animal Health and Internal Medicine, Faculty of Veterinary Medicine, University of Tehran, Tehran, Iran, Tel: 09379260882; Email: nalidadi@ut.ac.ir

Submitted: November 19, 2020

Approved: December 14, 2020

Published: December 15, 2020

How to cite this article: Alidadi N. A new applied protocol concerning COVID-19`s pandemic control: "Outdoor Access Approach" remodel of living for ensuring better ventilation. Arch Food Nutr Sci. 2020; 4: 025-028.

\section{DOI: 10.29328/journal.afns.1001024}

Copyright: ๑ 2020 Alidadi N. This is an open access article distributed under the Creative Commons Attribution License, which permits unrestricted use, distribution, and reproduction in any medium, provided the original work is properly cited.

Keywords: COVID-19; Pandemic; Control; Air ventilation; One health

Check for updates

OPEN ACCESS

\section{Introduction}

Currently, as a global community, we are facing the most severe infectious pandemic in the human`s history which it is unheard, unexperienced and unprecedented so far. COVID-19 or severe acute respiratory syndrome (SARS) coronavirus 2 (SARS-CoV-2) is a new emerging animal-originated infectious disease that is going on to spread [1,2]. Although several independent vaccine production projects are working ahead for combating the pandemic but it is obvious that it cannot be a sufficient umbrella that could protect billions of mans in a short term. Indeed, the current protocols are disappointing for their claimed capabilities to efficiently contain the pandemic. Pains-taking disinfection of streets, ways, and inside of public and private buildings by military and voluntary forces, general lockdowns of businesses and different social gatherings and aggregations such as education centers, music concerts, sport events and tournaments have providing only a transient suppression of the pandemic and, then again its cycling flare up! A comprehensive study on non-pharmaceutical interventions (NPI) - that are the only choices to implicate in the absence of any effective drugs and vaccines [3] - showed that disinfection and cleaning of public surfaces are the least effective whereas social distancing and travel restrictions are top ranked in all protocols in the world [4]. However, there are controversial considerations such as "Many months into the COVID-19 pandemic, the coronavirus is still spreading uncontrolled through the U.S. Public health authorities including the U.S. Centers for Disease Control and Prevention (CDC) and the World Health Organization (WHO) tell us to remain six feet apart, wash our hands, disinfect frequently touched surfaces, and wear masks. But compliance with these measures-especially masks-is mixed, and daily we hear of cases where people do not know how they were infected. 
We hear about superspreading events, where one person infects many, happening in crowded bars and family gatherings, but not at outdoor demonstrations. Beaches in cities like Chicago are closed, but gyms and indoor dining at restaurants have reopened. It is no wonder the public is confused" wrote a high ranked American expert, Dr. Jimenez [5]. Growing data are being evidenced that very light invisible micro-droplets of COVID-19 emitted by speaking, smiling and singing have crucial role in explosive and superspreading waves of the affection compared to the heavy macro-droplets produced by sneezing and coughing that the latter one have a ballistic behavior, and therefore are not so dangerously as they were assumed before. Various independent reports worldwide emphasize that the most of COVID-19' infections are rarely occurred in outdoor spaces [6-15]. There is mandatory to accept this reality as soon as possible that our protocols are disappointing for their delayed neglecting the importance of very dangerous micro-droplets of COVID-19 virus. One notable question is why many healthcare staff are unfortunately being infected and died by COVID-19 even while having the best personal protective equipment (PPE)??! Whereas the football's players are affected by COVID-19 in a very lower rates of quantity and severity compared to healthcare staff even though they are running rapidly and intensively side-by-side and face-to-face! So, it is a necessity to re-ordering of the protocols to highlight the vital impact of providing efficient fresh air for diluting and removing of COVID-19's micro-droplets. "Outdoor Access Approach" (OAA) is an integrated modelling to establish air refreshing in our ambient environment in combination with social distancing, covering face mask and hand cleaning. The object of this article is to discuss Outdoor Access Approach.

\section{Materials and methods}

It is important to notice that the Outdoor Access Approach (OAA) does not intend to deny and negate the approved WHO and CDC protocols for the control of COVID-19 but it is a new model for completing and deepening of them. Adaptation of all aspects of ordinary life activities in human societies to guarantee an efficient fresh air flow into enclosed spaces and prevention of the most dangerous air stagnation is the core stone of the OAA.

1- Opposite and cross windows and if possible entrance doors should be opened permanently in all private and public buildings and enclosed spaces of buses, taxies and cars to provide fresh air. For airplanes, there is a need to designation of proper potent ventilator and air-conditioner equipped by anti-coronal filters. The governments should program to adopt the majority of public activities to be carried out in outsides of building as possible.

2- Adjusted potent industrial air blowers and vacuum cleaners should be launched in any enclosed, crowded, busy and traveled places and spaces such as factories, airports, subway stations, hospitals, clinics, education centers including primary and high schools and universities, military bases, sport clubs, banks, hotels, malls, shops, restaurants, buses, trains and any kinds of businesses adopted to successful experiences of veterinary medical clinicians in health management of contagious air-borne respiratory diseases in enclosed animal farms.

3- Alongside to the using of air blowers and air vacuum cleaners, there is a need to air-conditioning by suitable adjusted fan-having equipment according to season, fan water-cooler or fan gas-burner heater (Figures 1-3). As, they can provide comfortable temperature and moisture in the ventilated ambient environment, and also help to prevent

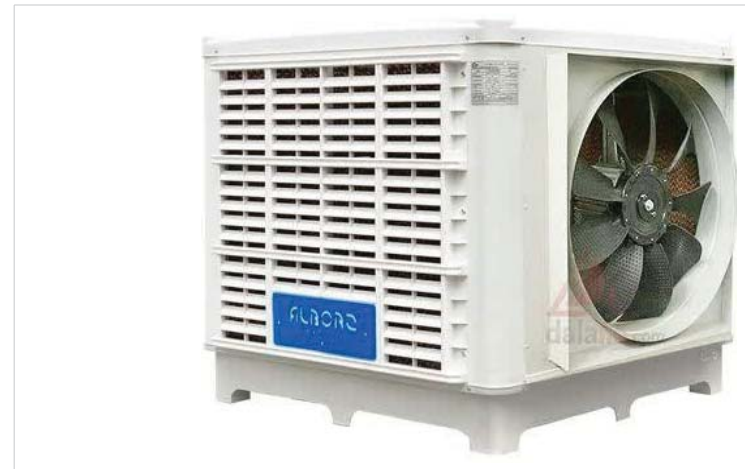

Figure 1: Fan evaporative, water-cooler for providing fresh air from outdoor to indoor spaces in summers.

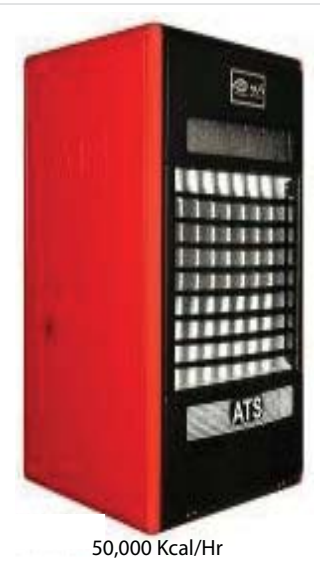

Figure 2: Fan gas-burner heater for providing fresh air from outdoor to indoor of public spaces in winters.

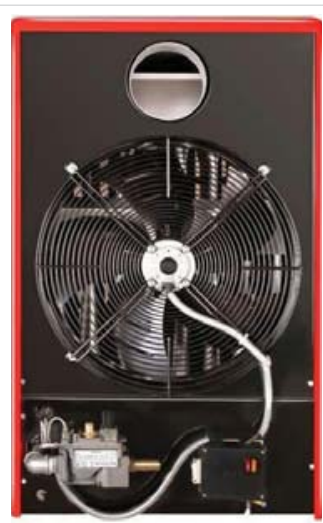

Figure 3: Fan gas-burner heater for providing fresh air from outdoor to indoor of public spaces in winters. 
air stagnation and, to make air dilution and removing of all microbial particles suspended in the ambient respiratory air including COVID-19 particles. Instead, closed-circuit central ventilation systems and air-conditioners should be turned off unless to be equipped by effective anti-coronaviral filters [16]. The gas-burner heaters have two kinds of energy-saving industrial and official, both of them bringing ambient air by a fan between hot heated blades and then throwing warmed air toward their front sides. This process of heating air can destroy microbial particles and to dilute and remove them where the cross windows and entrance doors are proportionally opened. Of-course official and also residential hermetic gas heaters that are smart, low consumption with double wall chimney have an advantage of being safe for classrooms.

\section{Discussion}

COVID-19 is a mucoso-respiratory disease that has showed very rapid transmission rate in the world that it could not attain to such a huge superspreading pandemic by so-called simple skin and face-to-face contacts between people [17]! Nasal cavity, oral cavity, eyes and ears are the routes of transmission and as another lipoprotein envelope virus i.e. rabies virus - which they have several noticeable similarities together- blood transfusion is not a usual way of transmission and both are labile to detergents such as water and soap washings and ethanol 70\%. However, in my years of veterinary medical experiences, disinfection of contaminated surfaces by enzymatic detergents such as home washing powders containing lipase and protease is safe, available, cost-effective and the most efficient compared to evaporable ethanol, inhalation-harmful sodium hypochlorite $(\mathrm{NaOCl})$ and the others for COVID-19.

Theoretically, COVID-19 virus is very labile to extreme hot and Scorcher climates like Khuzestan province of Iran. But this province experienced its peak of pandemic reaching to JuneJuly months compared to cold geographical mountainous locations that they were in a hypoepidemic stage! $[18,19]$ Because, the local people of southern Khuzestan ought to use close-circuit gas coolers and air-conditioners in completely enclosed buildings that may re-circulate the virus particles [16]. It is clear that the enclosed spaces have no sufficient access to fresh outdoor air, and so to have harmful recycling of contaminated air to COVID-19 virus.

Although, hand-washing with water and soap should be continued but it is never sufficient. Given insufficient air-refreshing and no access to outdoor in poor ventilated buildings, even physical distancing more than 10 meters with covering face mask is not effective to prevent new infections of COVID-19. The ordinary protocols are useful only to restrict reaching of large and heavy macro-droplets of COVID-19 produced by coughing, sneezing and speaking but cannot unfortunately obstruct entry of COVID-19's very light, smokelike micro-droplets which can be floated in air for minutes to hours [13,15,20-23] even using the most advanced personal protective equipment (PPE) as like as many of hospitals`staff involved in COVID-19 in throughout of the world.

Epidemiologic data indicate the majority of COVID-19 patients were infected in enclosed buildings that had no sufficient ventilation and access to outdoor spaces such as standing near opened windows and doors. Indeed, risk of infection in windy and sunny outdoors maintaining 2 meters' distance where covering face mask is negligible. Almost all confirmed COVID-19 cases had no access to outdoors. It is reported a case of outdoor transmission between two Chinese people occurring amongst 7000 cases involved in indoors. A population study showed that 6 percent of 25,000 COVID-119 cases were infected outdoors. It is interestingly that they were in intensive outdoor gatherings such as athletic events or musical concerts [6]. In almost all of 318 analyzed COVID-19 outbreaks, the infection transmitted to people in enclosed places [21]. The probability to be infected to COVID-19 in indoor places is 20 times more than in outdoor spaces [5]. So considering a large accumulated evidences it is rationale to say that more than $90 \%$ of the COVID-19 infections have been occurred in indoor spaces.

It is considered that $\mathrm{CO} 2$ concentration is an indicator of air exhalation and so an index of air stagnation which is linked to the likelihood of COVID-19 infection [24]. There is a relationship between poor ventilation and tuberculosis. Successful control of human and veterinary medical authorities to control of similar air-borne diseases i.e. influenza and SARS to be a life-threatening zoonotic pandemic shows that increasing air ventilation in enclosed locations along with the other health protocol are effective to contain the disease $[3,25,26]$.

Nevertheless, it is suggested that Outdoor Access Approach and using fan-equipment by diluting COVID-19 infective dose in the ambient inspiratory air in enclosed buildings is an applied effective modelling to prevent speed of transmission of the virus into mucosal membranes and its pathogenicity including respiratory tract. In conclusion it seems applying Outdoor Access Approach can return normal living activities with least mortality and morbidity rates if to be generally enrolled in all aspects of our daily life style. But, more research work is needed in this regard.

\section{References}

1. WHO. Coronavirus disease 2019 (COVID-19) situation reports. 2020. https://www.who.int/emergencies/diseases/novel-coronavirus-2019/ situation-reports

2. WHO-China. Report of the WHO-China joint mission on Coronavirus disease (Covid19). 2020.

3. Qualls N, Levitt A, Kanade N, Wright-Jegede N, Dopson S, et al. Community mitigation guidelines to prevent pandemic influenza. Morbidity and mortality report (MMWR). Recommendations and Report. 2017; 66: 1-34. 
4. Haug N, Geyrhofer L, Londei A, Dervic E, Desvars-Larrive A, et al. Ranking the effectiveness of worldwide COVID-19 government interventions. Nature Human Behaviour. 2020; 4: 1303-1312. PubMed: https://pubmed.ncbi.nlm.nih.gov/33199859/

5. Jimenez JL. COVID-19 is transmitted through aerosols. we have enough evidence, now it is time to act. (Dr. Jimenez is a fellow of Cooperative Institute for Research in Environmental Sciences at the University of Colorado-Boulder, the American Association for Aerosol Research and the American Geophysical Union). Time. 2020; 20.

6. AFP-JIJI. Catching coronavirus outside is rare but not impossible. 2020. www.japantimes.co.jp

7. CDC. Employer information for office buildings. 2020.

8. CDC. How protect yourself and others against covid19. 2020.

9. ECDC. Heating, ventilation, air-conditioning systems in the context of COVId-20: first update. Stockholm. 2020; 4.

10. Fears AC, Klimstra WB, Duprex $P$, et al. Comparative dynamic aerosol efficiencies of three emergent coronaviruses and the unusual persistence of SARS-CoV-2 in aerosol suspensions. medRxiv 2020.

11. Frieden TR, Lee CT. Identifying and interrupting superspreading events. Implications for control of Severe Acute Respiratory Syndrome Coronavirus2. Emerg Infect Dis. 2020; 26: 1059-1066.

PubMed: https://pubmed.ncbi.nlm.nih.gov/32187007/

12. GOV.UK. Public advice to cover faces in enclosed spaces. Department of Health and Social Care. 2020.

13. Morgenstern J. COVID-19 is spread by aerosols: an evidence review (2020): Updated December 2, 2020| First10EM Blog. 2020. https://first10em.com/COVID-19-is-spread-by-aerosols-an-evidencereview

14. Nishiura H, Oshitni $H$, Kobayashi $T$, Saito $T$, Sunagawa $T$, et al. Closed environments facilitate secondary transmission of coronavirus disease 2019 (COVID-19). medRxiv. 2020.

15. Watson J. Growing evidence COVID-19 spread by micro droplets. 2020. https://www.uwa.edu.au/news/article/2020/july
16. Chirico F, Sacco A, Bragazzi NL, Magnavita N. Can Air-Conditioning Systems Contribute to the Spread of SARS/MERS/COVID-19 Infection? Insights from a Rapid Review of the Literature. Int J Environ Res Public Health. 2020; 17: 6052: 1-11.

PubMed: https://pubmed.ncbi.nlm.nih.gov/32825303/

17. Hamner L, Dubbel P, Capron I, Ross A, Jordan A, et al. High SARSCoV-2 attack rate following exposure at a choir practice - Skagit County, Washington, March 2020. Morbidity and Mortality Weekly Report (MMWR). US Department of Health and Human Services. Centers for Disease Control and Prevention. 2020; 69: 605-610.

18. Erin C. Iran's coronavirus cases surge again in strategic province (Khuzestan) becomes new epicenter. 2020. Washingtonpost.com

19. Khuzestan`s Official state television. Another nurse dies; In Khuzestan (province), COVID-19 hit like a bomb. 2020. Women.ncr.iran.org

20. Li Y, Leung GM, Tang JW, Yang X, Chao CYH, et al. Role of ventilation in airborne transmission of infectious agents in the built environment? A multidisciplinary systematic review. Indoor Air. 2020; 17: 2-18. PubMed: https://pubmed.ncbi.nlm.nih.gov/17257148/

21. Qian H, Miao T, Li L, Zheng X, Luo D. et al. Indoor transmission of SARS-CoV-2. medRxiv. 2020.

22. van Doremalen $\mathrm{N}$, Bushmaker $\mathrm{T}$, Morris $\mathrm{DH}$. Aerosol and surface stability of SARS-CoV-2 as compared with SARS-CoV-1. New Eng J Med. 2020; 382: 1564-1567.

PubMed: https://pubmed.ncbi.nlm.nih.gov/32182409/

23. Yan Z, Lan Y. Modeling COVID-19 infection in a combined space. Nonlinear Dynamics. 2020; 101: 1643-1651.

24. Bhagat RK, Davies Wykes MS, Dalziel SB, Linden PF. Effects of ventilation on the indoor spread of COVID-19. Published online by Cambridge University Press: 2020.

25. Miller SL, Nazaroff WW, Jimenez JL, Boerstra A, Buonanno G, et al. Transmission of SARS-CoV-2 by inhalation of respiratory aerosol in the Skagit Valley Chorale superspreading event. medRxiv. 2020.

26. OIE report. Avian Influenza Portal. Control and prevention. $f$ ile:///E:/manuscript/Prevention and Control_ OIE - World Organisation for Animal Health.htm. Copyright $\odot$ OIE $20 \overline{2} 0$. 